\title{
Linearized Equations of General Relativity and the Problem of Reduction to the Newton Theory
}

\author{
Valery V. Vasiliev, Leonid V. Fedorov \\ Institute of Problems in Mechanics, Russian Academy of Sciences, Moscow, Russia \\ Email:vvvas@dol.ru
}

How to cite this paper: Vasiliev, V.V. and Fedorov, L.V. (2020) Linearized Equations of General Relativity and the Problem of Reduction to the Newton Theory. Journal of Modern Physics, 11, 221-236. https://doi.org/10.4236/jmp.2020.112014

Received: January 8, 2020

Accepted: February 10, 2020

Published: February 13, 2020

Copyright $\odot 2020$ by author(s) and Scientific Research Publishing Inc. This work is licensed under the Creative Commons Attribution International License (CC BY 4.0).

http://creativecommons.org/licenses/by/4.0/

(c) (i) Open Access

\begin{abstract}
The paper is concerned with the problem of reduction of the general relativity theory to the Newton gravitation theory for a gravitation field with relatively low intensity. This problem is traditionally solved on the basis of linearized equations of general relativity which, being matched to the Newton theory equations, allow us to link the classical gravitation constant with the constant entering the general relativity equations. Analysis of the linearized general relativity equations shows that it can be done only for empty space in which the energy tensor is zero. In solids, the set of linearized general relativity equations is not consistent and is not reduced to the Newton theory equations. Specific features of the problem are demonstrated with the spherically symmetric static problem of general relativity which has the closed-form solution.
\end{abstract}

\section{Keywords}

General Relativity, Gravitation Constant, Linearized Equations, Spherically Symmetric Problem

\section{Introduction. General Relativity Equations}

The basic equation of general relativity which specifies the Einstein tensor has the following form:

$$
E_{i}^{j}=R_{i}^{j}-\frac{1}{2} \delta_{i}^{j} R
$$

in which $R_{i}^{j} \quad\left(R=R_{i}^{i}\right)$ are the components of the Ricci curvature tensor (we use mixed components because for the spherically symmetric problem considered further they coincide with the physical components). The Einstein tensor is associated with the energy tensor as

$$
E_{i}^{j}=\chi T_{i}^{j}
$$


where $\chi$ is the relativity gravitational constant. The energy tensor expressed with the aid of Equations (1) and (2) identically satisfies the conservation equation

$$
\nabla_{k} T_{i}^{k}=0
$$

For the static problem,

$$
T_{i}^{j}=\sigma_{i}^{j}, T_{i}^{4}=0(i, j=1,2,3), T_{4}^{4}=\mu c^{2}
$$

where $\sigma_{i}^{j}$ is the stress tensor and $\mu$ is the density.

For gravitation with relatively low intensity, the general relativity must reduce to the Newton theory in which the gravitation potential $\psi$ satisfies the Poisson equation

$$
\Delta \psi=4 \pi G \mu
$$

in which $G$ is the classical gravitation constant. Traditionally, the linearized version of Equation (1) is obtained and matched to Equation (5). The result is

$$
\chi=\chi_{0}=8 \pi G / c^{4}
$$

\section{Linearized Equations and the Reduction Problem}

Consider the space referred to Cartesian coordinates $x^{i}(i=1,2,3)$ and present the line element of the Riemannian space as

$$
\mathrm{d} s^{2}=g_{i j} \mathrm{~d} x^{i} \mathrm{~d} x^{j}-g_{44} c^{2} \mathrm{~d} t^{2}
$$

Assume that the components of the metric tensor have the following forms: $g_{i j}=\delta_{i j}+f_{i j}$ and $g_{44}=1+f_{44}$ in which the amplitude values of functions $f$ are much smaller than unity. Undertaking linearization of Equation (1) and taking into account Equations (2) and (4), we arrive at the following set of the linearized general relativity equations:

$$
\begin{aligned}
\chi \sigma_{1}^{1} & =\frac{1}{2}\left(-f_{22,33}-f_{33,22}+2 f_{23,23}-f_{44,22}-f_{44,33}\right) \\
\chi \sigma_{1}^{2} & =\frac{1}{2}\left(f_{33,12}+f_{12,33}-f_{13,23}-f_{23,13}+f_{44,12}\right) \\
\chi \sigma_{1}^{3} & =\frac{1}{2}\left(f_{22,13}-f_{12,23}+f_{13,22}-f_{23,12}+f_{44,13}\right) \\
\chi \sigma_{2}^{2} & =\frac{1}{2}\left(-f_{11,33}-f_{33,11}+2 f_{13,13}-f_{44,11}-f_{44,33}\right) \\
\chi \sigma_{2}^{3} & =\frac{1}{2}\left(f_{11,23}-f_{12,13}-f_{13,12}+f_{23,11}+f_{44,23}\right) \\
\chi \sigma_{3}^{3} & =\frac{1}{2}\left(-f_{11,22}-f_{22,11}+2 f_{12,12}-f_{44,11}-f_{44,22}\right) \\
\chi \mu c^{2}=-\frac{1}{2}\left(f_{11,33}+f_{11,22}\right. & \left.+f_{22,11}+f_{22,33}+f_{33,11}+f_{33,22}-2 f_{12,12}-2 f_{13,13}-2 f_{23,23}\right)
\end{aligned}
$$

Notation $(\cdots)_{i}$ means the derivative with respect to $x^{i}$. The linearized form 
of the conservation equation, Equation (3), with regard to Equations (4) is

$$
\sigma_{1,1}^{1}+\sigma_{1,2}^{2}+\sigma_{1,3}^{3}-\frac{1}{2} f_{44,1}\left(\mu c^{2}-\sigma_{1}^{1}\right)+\frac{1}{2} f_{44,2} \sigma_{1}^{2}+\frac{1}{2} f_{44,3} \sigma_{1}^{3}=0 \quad(1,2,3)
$$

Here, $(1,2,3)$ means permutation which allows us to obtain two more equations from the written one (only three Equations (14) exist for a static problem). For linear approximation, we can neglect the nonlinear terms with stresses in Equation (14) and simplify it as

$$
\sigma_{1,1}^{1}+\sigma_{1,2}^{2}+\sigma_{1,3}^{3}-\frac{1}{2} f_{44,1} \mu c^{2}=0 \quad(1,2,3)
$$

This equation has a simple physical meaning-it is the equilibrium equation for a solid element loaded with gravitation forces.

Using the traditional way to derive Equation (6) for the gravitation constant, express the derivatives $f_{23,23}, f_{13,13}, f_{12,12}$ from Equations (7), (10), (12) and substitute them in Equation (13). The resulting equation is

$$
\Delta f_{44}=\chi\left(\mu c^{2}-\sigma\right)
$$

in which $\sigma=\sigma_{1}^{1}+\sigma_{2}^{2}+\sigma_{3}^{3}$ is the invariant of the stress tensor. For the linear approximation, we neglect $\sigma$ in comparison with $\mu c^{2}$ and finally get

$$
\Delta f_{44}=\chi \mu c^{2}
$$

Matching Equations (5) and (17), we can conclude that $f_{44}=2 \psi / c^{2}$ and $\chi=\chi_{0}$, where $\chi_{0}$ is specified by Equation (6). Thus, it looks like the general relativity reduces in the linear approximation to the Newton gravitation theory.

However, more careful analysis shows that the foregoing derivation is not correct for solids. The problem is in Equations (7)-(13) which are, in general, not compatible. As can be directly checked, the left-hand parts of these equations must satisfy the following relationship:

$$
\sigma_{1,1}^{1}+\sigma_{1,2}^{2}+\sigma_{1,3}^{3}=0 \quad(1,2,3)
$$

Consider first the empty space for which $\sigma_{i}^{j}=0$ and $\mu=0$. In this case, Equation (18) coincides with Equation (15) and the set of Equations (7)-(13) is compatible. Equation (17) is homogeneous and coincides with Equation (5) which is also homogeneous. Thus, for the empty space, the general relativity theory reduces to the Newton gravitation theory for gravitation with relatively low intensity. However, the situation is different in case of solids for which the equilibrium equation, Equation (15), must be satisfied. As follows from Equations (15) and (18), the equilibrium equations are satisfied only if $f_{44}=0$ which means the absence of gravitation. Thus, the linearized equations of general relativity do not describe gravitation and the linearized general relativity does not reduce to the Newton theory for solids. The reason is evident-whereas the terms with the stresses in Equation (15) are linear and refer to the first-order approximation, the last term with $f_{44}$ belongs to the second-order approximation. Naturally, this does not mean that the last term can be neglected-in this case, the gravitation disappears. This situation is not unique in mechanics of 
solids. For example, to construct the two-dimensional theory of thin plates from three-dimensional equations of theory of elasticity by the asymptotic method, we need to retain small terms of the first and of the second orders. Neglecting the second-order terms, we arrive at the plate theory which is not physically consistent [1]. Consider further the spherically symmetric problem for which closed-form solutions can be obtained.

\section{Spherically Symmetric Problem}

\subsection{Classical Linear Solution}

For comparison with the general relativity solutions that are discussed further, consider the problem of the theory of elasticity for a linear elastic isotropic solid sphere with radius $R$ and constant density $\mu$ loaded with gravitation forces following from the Newton theory. The gravitational potential $\psi$ is the solution of the Poisson equation

$$
\Delta \psi=\psi^{\prime \prime}+\frac{2}{r} \psi^{\prime}=4 \pi G \mu
$$

Here, $(\cdots)^{\prime}=\mathrm{d}(\cdots) / \mathrm{d} r$ and $r$ is the radial coordinate. For the external space ( $r \geq R$, index " $e$ "), $\mu=0$ and the solution of Equation (19) is $\psi_{e}=-G m / r$ in which $m$ is the sphere mass. Introduce the so-called gravitational radius

$$
r_{g}=\frac{2 G m}{c^{2}}
$$

Then, $\psi_{e}=-r_{g} c^{2} / 2 r$. For the internal space $(0 \leq r \leq R$, index “ $i$ "), the regular solution of Equation (19) is

$$
\psi_{i}=\frac{2}{3} \pi G \mu r^{2}+C
$$

Determining constant $C$ from the boundary condition $\psi_{i}(R)=\psi_{e}(R)$ and using Equation (20), we get

$$
\psi_{i}=-\frac{2}{3} \pi G \mu\left(R^{2}-r^{2}\right)-\frac{r_{g} c^{2}}{2 R}
$$

The equilibrium equation for the sphere under the action of gravitational body forces $f_{g}=-\mu \psi_{i}^{\prime}$ is

$$
\sigma_{r}^{\prime}+\frac{2}{r}\left(\sigma_{r}-\sigma_{\theta}\right)-\frac{4}{3} \pi G \mu^{2} r=0
$$

where $\sigma_{r}$ and $\sigma_{\theta}$ are the radial and the circumferential stresses. Consider the case of the perfect fluid for which $\sigma_{r}=\sigma_{\theta}=-p$. The pressure $p$ can be found from Equation (21) which takes the form

$$
p^{\prime}+\frac{4}{3} \pi G \mu^{2} r=0
$$

The solution of this equation that satisfies the boundary condition $p(r=R)=0$ is

$$
p=\frac{2}{3} \pi G \mu^{2}\left(R^{2}-r^{2}\right)
$$


In general relativity, the space geometry is Riemannian and the line element in spherical coordinates $r, \theta, \varphi$ is

$$
\mathrm{d} s^{2}=g_{11} \mathrm{~d} r^{2}+g_{22}\left(\mathrm{~d} \theta^{2}+\sin ^{2} \theta \mathrm{d} \varphi^{2}\right)-g_{44} c^{2} \mathrm{~d} t^{2}
$$

The components of the metric tensor depend on the radial coordinate only. For the foregoing linear solution, the general relativity interpretation of the obtained results is [2]

$$
g_{11}=1+\frac{r_{g}}{r}, \quad g_{22}=r^{2}, \quad g_{44}=1-\frac{r_{g}}{r}
$$

In case $r_{g}=0$, the space is Euclidean and gravitation vanishes.

\subsection{General Relativity Equations}

For a spherically symmetric problem, the field equations following from Equation (1) reduce to [3]

$$
\begin{gathered}
E_{1}^{1}=\frac{1}{g_{22}}-\frac{1}{g_{11}}\left[\frac{1}{4}\left(\frac{g_{22}^{\prime}}{g_{22}}\right)^{2}+\frac{g_{22}^{\prime} g_{44}^{\prime}}{2 g_{22} g_{44}}\right] \\
E_{2}^{2}=-\frac{1}{2 g_{11}}\left[\frac{g_{44}^{\prime \prime}}{g_{44}}-\frac{1}{2}\left(\frac{g_{44}^{\prime}}{g_{44}}\right)^{2}+\frac{g_{22}^{\prime \prime}}{g_{22}}-\frac{1}{2}\left(\frac{g_{22}^{\prime}}{g_{22}}\right)^{2}+\frac{g_{22}^{\prime}}{2 g_{22}}\left(\frac{g_{44}^{\prime}}{g_{44}}-\frac{g_{11}^{\prime}}{g_{11}}\right)-\frac{g_{11}^{\prime} g_{44}^{\prime}}{2 g_{11} g_{44}}\right] \\
E_{4}^{4}=\frac{1}{g_{22}}-\frac{1}{g_{11}}\left[\frac{g_{22}^{\prime \prime}}{g_{22}}-\frac{1}{4}\left(\frac{g_{22}^{\prime}}{g_{22}}\right)^{2}-\frac{g_{11}^{\prime} g_{22}^{\prime}}{2 g_{11} g_{22}}\right]
\end{gathered}
$$

in which in accordance with Equations (2) and (4)

$$
E_{1}^{1}=\chi T_{1}^{1}=\chi \sigma_{r}, \quad E_{2}^{2}=\chi T_{2}^{2}=\chi \sigma_{\theta}, \quad E_{4}^{4}=\chi T_{4}^{4}=\chi \mu c^{2}
$$

The only one conservation equation, Equation (3), becomes

$$
\left(T_{1}^{1}\right)^{\prime}+\frac{g_{22}^{\prime}}{g_{22}}\left(T_{1}^{1}-T_{2}^{2}\right)+\frac{g_{44}^{\prime}}{2 g_{44}}\left(T_{1}^{1}-T_{4}^{4}\right)=0
$$

The solution of the external $(r \geq R)$ problem must satisfy the asymptotic conditions and to reduce to Equations (25) for $r \rightarrow \infty$. The solution for the internal $(0 \leq r \leq R)$ problem must satisfy the symmetry condition at the sphere center according to which $g_{11}(0)=1, g_{22}(0)=0$. Both solutions must satisfy the boundary conditions on the sphere surface, i.e.

$$
g_{11}^{e}(R)=g_{11}^{i}(R), \quad g_{22}^{e}(R)=g_{22}^{i}(R), \quad g_{44}^{e}(R)=g_{44}^{i}(R)
$$

Substitution of the obtained equations for $T_{i}^{i}$ in Equation (30) identically satisfies this equation. So, only three of four Equations (26)-(28) and (30) are mutually independent. Traditionally [3], the simplest set of equations including Equations (26), (28) and (30) is used. The obtained solution identically satisfies Equation (27). To solve the problem, we should supplement Equations (26), (28) and (30) which include three components of the metric tensor $g_{11}, g_{22}, g_{44}$ and two stresses $\sigma_{r}, \sigma_{\theta}$ with one coordinate condition for the metric tensor [4] and one equation for the stresses [5]. For the case of perfect fluid which is considered 
further, $\sigma_{r}=\sigma_{\theta}=-p$ and we have four unknown functions and need only one coordinate condition for the metric tensor.

\subsection{Linearized Solution}

Decompose the components of the metric tensor in Equation (24) as $g_{11}=1+f_{1}$, $g_{22}=r^{2}\left(1+f_{2}\right), \quad g_{44}=1+f_{4}$ and assume that the absolute values of functions $f(r)$ are much less than unity. Undertaking linearization of Equations (26)-(28), we arrive at

$$
\left(E_{1}^{1}\right)_{1}=\frac{1}{r^{2}}\left(f-r f_{4}^{\prime}\right),\left(E_{2}^{2}\right)_{1}=\frac{1}{2 r}\left(f-r f_{4}^{\prime}\right)^{\prime}, \quad\left(E_{4}^{4}\right)_{1}=\frac{1}{r^{2}}(r f)^{\prime}
$$

where $f=f_{1}-\left(r f_{2}\right)^{\prime}$. Using Equations (29), we can present these equations as

$$
\chi \sigma_{r}=\frac{1}{r^{2}}\left(f-r f_{4}^{\prime}\right), \quad \chi \sigma_{\theta}=\frac{1}{2 r}\left(f-r f_{4}^{\prime}\right)^{\prime}, \quad \chi \mu c^{2}=\frac{1}{r^{2}}(r f)^{\prime}
$$

The conservation equation, Equation (30), transformed with aid of Equation (29) becomes

$$
\sigma_{r}^{\prime}+\frac{2}{r}\left(\sigma_{1}-\sigma_{2}\right)+\frac{1}{2} f_{4}^{\prime}\left(\sigma_{r}-\mu c^{2}\right)=0
$$

Neglecting $\sigma_{r}$ in comparison with $\mu c^{2}$ in the last term of this equation, we can conclude that it is analogous to the equilibrium equation, Equation (21).

Proceeding, express $f$ from the first equation of Equation (33), $f^{\prime}$ from the second of these equations, i.e.,

$$
f=r^{2} \chi \sigma_{r}+r f_{4}^{\prime}, \quad f^{\prime}=2 r \chi \sigma_{\theta}+f_{4}^{\prime}+r f_{4}^{\prime \prime}
$$

and substitute in the third equation to get

$$
f_{4}^{\prime \prime}+\frac{2}{r} f_{4}^{\prime}=\chi\left(\mu c^{2}-\sigma\right)
$$

in which $\sigma=\sigma_{r}+2 \sigma_{\theta}$ is the invariant of the stress tensor. Neglecting $\sigma$ in comparison with $\mu c^{2}$, we arrive at

$$
\Delta f_{4}=\chi \mu c^{2}
$$

Formally, taking $f_{4}=2 \psi / c^{2}$ and $\chi=8 \pi G / c^{4}$, we can reduce this equation to the Newton theory equation, Equation (19). However, as in Section 2, the foregoing derivation is not correct. For the external space $\sigma_{r}=\sigma_{\theta}=0$ and $\mu=0$. Both Equations (19) and (35) are homogeneous and the linearized general relativity reduces to the Newton theory. Taking $\sigma_{r}=\sigma_{\theta}=0$ in Equations (33), we can conclude that the second equation is a derivative of the first one. Thus we have only two independent Equations (33) for three functions $f_{1,2,4}$ and need a coordinate condition to find these functions. For the internal space, the first two Equations (33) have different left-hand parts and include one unknown function $F=f-r f_{4}^{\prime}$ in the right-hand parts. This means that, in general, these equations are not compatible. To derive the compatibility condition, express $F$ from the first equation and substitute in the second one. The resulting equation is 


$$
\sigma_{r}^{\prime}+\frac{2}{r}\left(\sigma_{r}-\sigma_{\theta}\right)=0
$$

This equation coincides with the equilibrium equation, Equation (21) only in the absence of gravitation which is, naturally, not the case. Thus, the linearized equations of general relativity are not compatible for a solid sphere and to arrive at the consistent theory, we need to construct the second-order approximation.

\subsection{Second-Order Asymptotic Approximation}

Present the components of the metric tensor entering Equation (24) as

$$
g_{11}=1+\varepsilon f_{1}+\varepsilon^{2} \varphi_{1}, \quad g_{22}=r^{2}\left(1+\varepsilon f_{2}+\varepsilon^{2} \varphi_{2}\right), \quad g_{44}=1+\varepsilon f_{4}+\varepsilon^{2} \varphi_{4}
$$

in which $\varepsilon$ is a small parameter. Substitution in Equation (1) yields

$$
E_{i}^{i}=\varepsilon\left(E_{i}^{i}\right)_{1}+\varepsilon^{2}\left(E_{i}^{i}\right)_{2}
$$

Here, the components $\left(E_{i}^{i}\right)_{1}$ are specified by Equations (32) and

$$
\begin{gathered}
\left(E_{1}^{1}\right)_{2}=\frac{1}{r^{2}}\left(\varphi_{1}-\varphi_{2}+f_{2}^{2}-f_{1}^{2}\right) \\
+\frac{1}{r}\left[-\varphi_{2}^{\prime}-\varphi_{4}^{\prime}+\left(f_{1}+f_{4}\right) f_{4}^{\prime}+\left(f_{1}+f_{2}\right) f_{2}^{\prime}\right] \\
+\frac{f_{2}^{\prime}}{4}\left(f_{2}^{\prime}+2 f_{4}^{\prime}\right) \\
\left(E_{2}^{2}\right)_{2}=\frac{1}{2 r}\left[\varphi_{1}^{\prime}-2 \varphi_{2}^{\prime}-\varphi_{4}^{\prime}+\left(f_{1}+f_{4}\right) f_{4}^{\prime}+2\left(f_{1}+f_{2}\right) f_{2}^{\prime}-2 f_{1} f_{1}^{\prime}\right] \\
+\frac{1}{4}\left[-2\left(\varphi_{2}^{\prime \prime}+\varphi_{4}^{\prime \prime}\right)+\left(f_{2}^{\prime}\right)^{2}+\left(f_{4}^{\prime}\right)^{2}+2\left(f_{1} f_{2}^{\prime \prime}+f_{1} f_{4}^{\prime \prime}+f_{2} f_{2}^{\prime \prime}+f_{4} f_{4}^{\prime \prime}\right)\right. \\
\left.+f_{1}^{\prime} f_{2}^{\prime}+f_{1}^{\prime} f_{4}^{\prime}-f_{2}^{\prime} f_{4}^{\prime}\right] \\
\left(E_{4}^{4}\right)_{2}=\frac{1}{r^{2}}\left(\varphi_{1}-\varphi_{2}-f_{1}^{2}+f_{2}^{2}\right)+\frac{1}{r}\left(\varphi_{1}^{\prime}-3 \varphi_{2}^{\prime}-2 f_{1} f_{1}^{\prime}+3 f_{1} f_{2}^{\prime}+3 f_{2} f_{2}^{\prime}\right) \\
+\frac{1}{4}\left[-4 \varphi_{2}^{\prime \prime}+2 f_{1}^{\prime} f_{2}^{\prime}+\left(f_{2}^{\prime}\right)^{2}+4\left(f_{1}+f_{2}\right) f_{2}^{\prime \prime}\right]
\end{gathered}
$$

Recall that the Einstein tensor $E_{i}^{i}$ must satisfy the conservation equation, Equation (30), which can be written as

$$
\left(E_{1}^{1}\right)^{\prime}+\frac{g_{22}^{\prime}}{g_{22}}\left(E_{1}^{1}-E_{2}^{2}\right)+\frac{g_{44}^{\prime}}{2 g_{44}}\left(E_{1}^{1}-E_{4}^{4}\right)=0
$$

Substituting expressions (36) for the metric tensor, we arrive at the following two equations corresponding to $\varepsilon$ and $\varepsilon^{2}$ :

$$
\begin{gathered}
\left(E_{1}^{1}\right)_{1}^{\prime}+\frac{2}{r}\left[\left(E_{1}^{1}\right)_{1}-\left(E_{2}^{2}\right)_{1}\right]=0 \\
\left(E_{1}^{1}\right)_{2}^{\prime}+\frac{2}{r}\left[\left(E_{1}^{1}\right)_{2}-\left(E_{2}^{2}\right)_{2}\right]+f_{2}^{\prime}\left[\left(E_{1}^{1}\right)_{1}-\left(E_{2}^{2}\right)_{1}\right]+\frac{1}{2} f_{4}^{\prime}\left[\left(E_{1}^{1}\right)_{1}-\left(E_{4}^{4}\right)_{1}\right]=0
\end{gathered}
$$

Substituting Equations (32) and (38), we can conclude that both equations, Equations (39) and (40), are satisfied identically. Thus, the second approximation, in contrast to the first one discussed in Section (2.3), is consistent. This 
means that only two of three Equations (38) are mutually independent and we can proceed using only two equations, namely those for $E_{1}^{1}$ and $E_{4}^{4}$. However, these two equations include three unknown functions with indices 1,2 and 4. To solve the problem, we need to supplement the aforementioned two equations with a coordinate condition. According to the condition used further, the so-called space density $d=\sqrt{g_{R} / g_{E}}$ in which $g_{R}$ and $g_{E}$ are the determinants of the metric tensor in Riemannian and Euclidean three-dimensional spaces is minimized [5]. For the spherically symmetric problem, the introduced coordinate condition has the form [5]

$$
\sqrt{g_{11}} g_{22}=r^{2}
$$

For the internal space, this condition has a simple physical meaning - gravitation transforming the Euclidean geometry into Riemannian does not affect the volume element and the sphere mass, i.e.,

$$
m=4 \pi \mu \int_{0}^{R} \sqrt{g_{11}} g_{22} \mathrm{~d} r=4 \pi \mu \int_{0}^{R} r^{2} \mathrm{~d} r=\frac{4}{3} \pi \mu R^{3}
$$

So, the sphere mass is the same that in Euclidean space [6].

Using Equations (36), we can write Equation (41) as

$$
\varepsilon\left(\frac{f_{1}}{2}+f_{2}\right)+\varepsilon^{2}\left(\frac{\varphi_{1}}{2}+\varphi_{2}-\frac{f_{1}^{2}}{8}+\frac{f_{1} f_{2}}{2}\right)=0
$$

and get two coordinate conditions for the first and the second approximations, i.e.,

$$
f_{1}=-2 f_{2}, \varphi_{1}=3 f_{2}^{2}-2 \varphi_{2}
$$

Taking into account these results, we can transform Equations (32) and (38) for $E_{1}^{1}$ and $E_{4}^{4}$ to

$$
\begin{gathered}
\left(E_{1}^{1}\right)_{1}=-\frac{1}{r^{2}}\left(3 f_{2}+r f_{2}^{\prime}+r f_{4}^{\prime}\right) \\
\left(E_{4}^{4}\right)_{1}=-\frac{1}{r^{2}}\left[r\left(3 f_{2}+r f_{2}^{\prime}\right)\right]^{\prime} \\
\left(E_{1}^{1}\right)_{2}=-\frac{3 \varphi_{2}}{r^{2}}-\frac{1}{r}\left(\varphi_{2}^{\prime}+\varphi_{4}^{\prime}+f_{2} f_{2}^{\prime}+2 f_{2} f_{4}^{\prime}-f_{4} f_{4}^{\prime}\right)-\frac{1}{4}\left(f_{2}^{\prime}+2 f_{4}^{\prime}\right) f_{2}^{\prime} \\
\left(E_{4}^{4}\right)_{2}=-\left[\varphi_{2}^{\prime \prime}+\frac{3}{r^{2}} \varphi_{2}+\frac{5}{r}\left(\varphi_{2}^{\prime}+f_{2} f_{2}^{\prime}\right)+\frac{3}{4}\left(f_{2}^{\prime}\right)^{2}+f_{2} f_{2}^{\prime \prime}\right]
\end{gathered}
$$

Consider the external space $(R \leq r<\infty)$ for which $\left(E_{i}^{i}\right)_{1}=\left(E_{i}^{i}\right)_{2}=0$. Integration of Equation (45) yields

$$
r\left(f_{2}^{e}\right)^{\prime}+3 f_{2}^{e}=-\frac{C_{1}}{r}
$$

Further integration and the first equation of Equations (43) give

$$
f_{2}^{e}=-\frac{C_{1}}{2 r}+\frac{C_{2}}{r^{3}}, \quad f_{1}^{e}=\frac{C_{1}}{r}-\frac{2 C_{2}}{r^{3}}
$$


Applying Equation (44) and using Equations (48), we get

$$
r\left(f_{4}^{e}\right)^{\prime}=-3 f_{2}^{e}-r\left(f_{2}^{e}\right)^{\prime}=\frac{C_{1}}{r}
$$

Thus,

$$
f_{4}^{e}=-\frac{C_{1}}{r}
$$

Transforming Equation (47) with the aid of Equations (48), we have

$$
r^{2}\left(\varphi_{2}^{e}\right)^{\prime \prime}+5 r\left(\varphi_{2}^{e}\right)^{\prime}+3 \varphi_{2}^{e}=\frac{9 C_{1}^{2}}{16 r^{2}}-\frac{15 C_{2}^{2}}{4 r^{6}}-\frac{3 C_{1} C_{2}}{4 r^{4}}
$$

This is the Euler equation whose solution is

$$
\varphi_{2}^{e}=\frac{C_{3}}{r}+\frac{C_{4}}{r^{3}}-\frac{9 C_{1}^{2}}{16 r^{2}}-\frac{C_{2}^{2}}{4 r^{6}}-\frac{C_{1} C_{2}}{4 r^{4}}
$$

From the second equation of Equations (43) it follows that

$$
\varphi_{1}^{e}=-\frac{2 C_{3}}{r}-\frac{2 C_{4}}{r^{3}}+\frac{15 C_{1}^{2}}{8 r^{2}}+\frac{7 C_{2}^{2}}{2 r^{6}}-\frac{5 C_{1} C_{2}}{2 r^{4}}
$$

Using Equation (46) and taking into account Equations (48)-(50), we get

$$
\left(\varphi_{4}^{e}\right)^{\prime}=-\frac{2 C_{3}}{r^{2}}-\frac{C_{1} C_{2}}{r^{5}}
$$

Integration yields

$$
\varphi_{4}^{e}=\frac{2 C_{3}}{r}+\frac{C_{1} C_{2}}{4 r^{4}}+C_{5}
$$

Substituting Equations (48)-(52) in Equations (36), we arrive at the following expressions for the components of the metric tensor in the external space:

$$
\begin{gathered}
g_{11}^{e}=1+\varepsilon_{e}\left(\frac{C_{1}}{r}-\frac{2 C_{2}}{r^{3}}\right)+\varepsilon_{e}^{2}\left(-\frac{2 C_{3}}{r}-\frac{2 C_{4}}{r^{3}}+\frac{15 C_{1}^{2}}{8 r^{2}}+\frac{7 C_{2}^{2}}{2 r^{6}}-\frac{5 C_{1} C_{2}}{2 r^{4}}\right) \\
g_{22}^{e}=r^{2}\left[1+\varepsilon_{e}\left(-\frac{C_{1}}{2 r}+\frac{C_{2}}{r^{3}}\right)+\varepsilon_{e}^{2}\left(\frac{C_{3}}{r}+\frac{C_{4}}{r^{3}}-\frac{9 C_{1}^{2}}{16 r^{2}}-\frac{C_{2}^{2}}{4 r^{6}}-\frac{C_{1} C_{2}}{4 r^{4}}\right)\right] \\
g_{44}^{e}=1-\varepsilon_{e} \frac{C_{1}}{r}+\varepsilon_{e}^{2}\left(\frac{2 C_{3}}{r}+\frac{C_{1} C_{2}}{4 r^{4}}+C_{5}\right)
\end{gathered}
$$

For $r \rightarrow \infty$, the asymptotic behavior of the metric tensor is specified by Equations (25). Matching the obtained solution to these equations, we can conclude that $C_{1}=1, C_{3}=C_{5}=0$ and $\varepsilon_{e}=r_{g}$. Thus, for the external space, parameter $\varepsilon_{e}$ is equal to the gravitational radius in Equation (20) and the solution becomes

$$
\begin{gathered}
g_{11}^{e}=1+r_{g}\left(\frac{1}{r}-\frac{2 C_{2}}{r^{3}}\right)+r_{g}^{2}\left(-\frac{2 C_{4}}{r^{3}}+\frac{15}{8 r^{2}}+\frac{7 C_{2}^{2}}{2 r^{6}}-\frac{5 C_{2}}{2 r^{4}}\right) \\
g_{22}^{e}=r^{2}\left[1+r_{g}\left(-\frac{1}{2 r}+\frac{C_{2}}{r^{3}}\right)+r_{g}^{2}\left(\frac{C_{4}}{r^{3}}-\frac{9}{16 r^{2}}-\frac{C_{2}^{2}}{4 r^{6}}-\frac{C_{2}}{4 r^{4}}\right)\right]
\end{gathered}
$$




$$
g_{44}^{e}=1-\frac{r_{g}}{r}+r_{g}^{2} \frac{C_{2}}{4 r^{4}}
$$

The integration constants that enter this solution should be found from the boundary conditions on the sphere surface. To formulate the boundary conditions, we need to consider the internal $(0 \leq r \leq R)$ problem. Assume that the sphere consists of the perfect fluid for which $\sigma_{r}=\sigma_{\theta}=-p$. Thus, $E_{1}^{1}=E_{2}^{2}=-\chi p$ and $E_{4}^{4}=\chi \mu c^{2}$. In addition to Equations (36) and (37), decompose the pressure as

$$
p=\varepsilon_{i} p_{1}+\varepsilon_{i}^{2} p_{2}
$$

Then, we should take in Equations (37)

$$
\left(E_{1}^{1}\right)_{1}=\left(E_{2}^{2}\right)_{1}=-\chi p_{1},\left(E_{1}^{1}\right)_{2}=\left(E_{2}^{2}\right)_{2}=-\chi p_{2}
$$

Substituting the first of these equations in Equation (39), we get $p_{1}^{\prime}=0$. Taking into account the boundary condition for the pressure $p_{1}(r=R)=0$, we can conclude that $p_{1}=0$. This result explains the problem discussed in Section 2.3 in connection with the linearized solution. The pressure corresponding to this solution is zero which means that the linearized solution does not describe gravitation in solids.

To proceed, specify the parameter $\varepsilon_{i}$ for the internal problem. Assume that $\varepsilon_{i}=\chi \mu c^{2}$. Then, the corresponding equation in Equations (37) yields

$$
\left(E_{4}^{4}\right)_{1}=1,\left(E_{4}^{4}\right)_{2}=0
$$

Then, Equation (45) becomes

$$
\left\{r\left[3 f_{2}^{i}+r\left(f_{2}^{i}\right)^{\prime}\right]\right\}^{\prime}=-r^{2}
$$

The solution of this equation is

$$
f_{2}^{i}=-\frac{r^{2}}{15}+\frac{B_{1}}{r}+\frac{B_{2}}{r^{3}}
$$

This solution is regular at the sphere center $r=0$ if $B_{1}=B_{2}=0$. Using the first equation of Equations (43), we get

$$
f_{2}^{i}=-\frac{r^{2}}{15}, \quad f_{1}^{i}=\frac{2 r^{2}}{15}
$$

Taking $p_{1}=0$ in Equations (57) and applying Equation (44), we arrive at

$$
3 f_{2}^{i}+r\left(f_{2}^{i}\right)^{\prime}+r\left(f_{4}^{i}\right)^{\prime}=0
$$

Substituting the first equation of Equations (59) and integrating, we have

$$
f_{4}^{i}=\frac{1}{6}\left(r^{2}+B_{3}\right)
$$

Now, we can find the pressure in the fluid. Recall that $p_{1}=0$, so that

$$
p=\varepsilon_{i}^{2} p_{2}=\chi^{2} \mu^{2} c^{4} p_{2}
$$

Here, $p_{2}$ can be determined from Equation (40) which can be transformed with the aid of Equations (57), (58) and (60) to 


$$
\chi p_{2}^{\prime}+\frac{r}{6}=0
$$

The solution of this equation which satisfies the boundary condition $p_{2}(r=R)=0$ is

$$
p_{2}=\frac{1}{12 \chi}\left(R^{2}-r^{2}\right)
$$

Substituting this result in Equation (61), we finally get

$$
p=\frac{\chi \mu^{2} c^{4}}{12}\left(R^{2}-r^{2}\right)
$$

This solution must coincide with Equation (23) corresponding to the Newton theory. Matching Equations (23) and (62), we can conclude that $\chi=\chi_{0}$, where $\chi_{0}$ is given by Equation (6). Thus, Equation (6) is proved for the second-order asymptotic solution for the spherically symmetric problem. Using the obtained result and applying Equations (19) and (42) for $r_{g}$ and $m$, we can obtain the final form for parameter $\varepsilon_{i}$, i.e.,

$$
\varepsilon_{i}=\chi \mu c^{2}=\frac{3 r_{g}}{R^{3}}
$$

Thus, for the internal problem, the parameter is also expressed in terms of the gravitational radius.

Determine the metric tensor components for the second-order approximation. Consider Equation (47) whose left-hand part is zero because of Equations (58). Substituting the first equation of Equations (59), we obtain the following equation:

$$
\left(\varphi_{2}^{i}\right)^{\prime \prime}+\frac{5}{r}\left(\varphi_{2}^{i}\right)^{\prime}+\frac{3}{r^{2}} \varphi_{2}^{i}=-\frac{r^{2}}{15}
$$

The solution of this equation is

$$
\varphi_{2}^{i}=-\frac{r^{4}}{525}+\frac{B_{4}}{r}+\frac{B_{5}}{r^{3}}
$$

Using the regularity condition and the second equation in Equations (43), we get $B_{4}=B_{5}=0$ and

$$
\varphi_{2}^{i}=-\frac{r^{4}}{525}, \quad \varphi_{1}^{i}=\frac{3 r^{4}}{175}
$$

The function $\varphi_{4}^{i}(r)$ can be found from Equation (46) in which $\left(E_{1}^{1}\right)_{2}=-\chi p_{2}$. Using Equations (59), (60), (62) and (64) and integrating, we find

$$
\varphi_{4}^{i}=\frac{1}{60}\left[r^{2}\left(\frac{r^{2}}{4}+\frac{5}{2} R^{2}+\frac{5}{3} B_{3}\right)+B_{6}\right]
$$

Finally substituting Equations (59), (60), (64) and (65) in Equations (36) and using Equation (63) for $\varepsilon_{i}$, we arrive at the following expressions for the metric tensor components of the internal space:

$$
g_{11}^{i}=1+\frac{2 r_{g} r^{2}}{5 R^{3}}+\frac{27 r_{g}^{2} r^{4}}{175 R^{6}}
$$




$$
\begin{gathered}
g_{22}^{i}=r^{2}\left(1-\frac{r_{g} r^{2}}{5 R^{3}}-\frac{3 r_{g}^{2} r^{4}}{175 R^{6}}\right) \\
g_{44}^{i}=1+\frac{r_{g}}{2 R^{3}}\left(r^{2}+B_{3}\right)+\frac{3 r_{g}^{2}}{20 R^{6}}\left[r^{2}\left(\frac{r^{2}}{4}+\frac{5}{2} R^{2}+\frac{5}{3} B_{3}\right)+B_{6}\right]
\end{gathered}
$$

Determine the integration constants using the boundary conditions in Equations (31). Equating the terms with $r_{g}$ in Equations (53) and (66) and doing the same for the terms including $r_{g}^{2}$, we get

$$
C_{2}=\frac{3}{10} R^{2}, B_{3}=-3 R^{2}, C_{4}=\frac{9 R}{14}, B_{6}=\frac{11}{4} R^{4}
$$

Thus, the boundary conditions (31) for $g_{11}$ and $g_{44}$ are satisfied. The boundary condition for $g_{22}$ is satisfied because of Equation (41). Finally, we arrive at the following expressions for the components of the metric tensor in the external and internal spaces:

$$
\begin{gathered}
g_{11}^{e}=1+\bar{r}_{g}\left(\frac{1}{\bar{r}}+\frac{3}{5 \bar{r}^{3}}\right)+\bar{r}_{g}^{2}\left(\frac{15}{8 \bar{r}^{2}}-\frac{9}{7 \bar{r}^{3}}-\frac{3}{8 \bar{r}^{4}}+\frac{63}{200 \bar{r}^{6}}\right) \\
g_{22}^{e}=r^{2}\left[1+\bar{r}_{g}\left(-\frac{1}{2 \bar{r}}+\frac{3}{10 \bar{r}^{3}}\right)+9 \bar{r}_{g}^{2}\left(-\frac{1}{16 \bar{r}^{2}}+\frac{1}{14 \bar{r}^{3}}-\frac{1}{120 \bar{r}^{4}}-\frac{1}{400 \bar{r}^{6}}\right)\right] \\
g_{44}^{e}=1-\frac{\bar{r}_{g}}{\bar{r}}+\frac{3 \bar{r}_{g}^{2}}{40 \bar{r}^{4}} \\
g_{11}^{i}=1+\frac{2}{5} \bar{r}_{g} \bar{r}^{2}+\frac{27}{175} \bar{r}_{g}^{2} \bar{r}^{6} \\
g_{22}^{i}=r^{2}\left(1-\frac{1}{5} \bar{r}_{g} \bar{r}^{2}-\frac{3}{175} \bar{r}_{g}^{2} \bar{r}^{4}\right) \\
\bar{r}_{44}^{i}=1+\frac{3 \bar{r}_{g}^{2}}{2}\left(\bar{r}^{2}-3\right)+\frac{\left.\bar{r}^{2}\left(\bar{r}^{2}-10\right)+11\right]}{80}\left[\begin{array}{c}
2 \\
g^{2}
\end{array}\right.
\end{gathered}
$$

Here,

$$
\bar{r}=\frac{r}{R}, \quad \bar{r}_{g}=\frac{r_{g}}{R}
$$

For real objects, the ratio $\bar{r}_{g}$ is rather small. For example, for Earth $\bar{r}_{g}=1.4 \times 10^{-6}$, for Sun $\bar{r}_{g}=4.25 \times 10^{-6}$, for the largest of the observed visible stars-red supergiant UI Scutti $\left(R=11.9 \times 10^{11} \mathrm{~m}, \quad m=64 \times 10^{30} \mathrm{~kg}\right), \bar{r}_{g}=8 \times 10^{-9}$ [7].

Thus, the classical expression for the gravitational constant in Equation (6) is proved, but under the following conditions:

- The problem is spherically symmetric and static.

- The sphere consists of a perfect fluid with constant density.

- The asymptotic equations are of the second order, not of the first.

- The coordinate condition in Equation (41) is valid.

If the last of these conditions is violated, the result can be different. For example, take the coordinate condition in the form $g_{22}=r^{2}$ or $f_{2}=\varphi_{2}=0$ which 
corresponds to the Schwarzchild solution of the spherically symmetric static problem for a fluid sphere [3]. Using decompositions in Equations (36) and (37) under the conditions $f_{2}=\varphi_{2}=0$, we arrive at the following equations analogous to Equations (44)-(47):

$$
\begin{gathered}
\left(E_{1}^{1}\right)_{1}=\frac{1}{r^{2}}\left(f_{1}-r f_{4}^{\prime}\right) \\
\left(E_{4}^{4}\right)_{1}=\frac{1}{r^{2}}\left(r f_{1}\right)^{\prime} \\
\left(E_{1}^{1}\right)_{2}=\frac{1}{r^{2}}\left(\varphi_{1}-f_{1}^{2}\right)-\frac{\varphi_{4}^{\prime}}{r}+\frac{f_{4}^{\prime}}{r}\left(f_{1}+f_{4}\right) \\
\left(E_{4}^{4}\right)_{2}=\frac{1}{r^{2}}\left[r\left(\varphi_{1}-f_{1}^{2}\right)\right]^{\prime}
\end{gathered}
$$

Consider the external problem for which $E_{i}^{i}=0$. Integration of Equations (71) and (73) yields

$$
f_{1}^{e}=\frac{C_{1}}{r}, \varphi_{1}^{e}=\left(\frac{C_{1}}{r}\right)^{2}
$$

Using Equations (74), we can transform Equations (70) and (72) to

$$
\left(f_{4}^{e}\right)^{\prime}=\frac{C_{1}}{r^{2}},\left(\varphi_{4}^{e}\right)^{\prime}=\left(f_{1}^{e}+f_{4}^{e}\right)\left(f_{4}^{e}\right)^{\prime}
$$

Integration gives

$$
f_{4}^{e}=-\frac{C_{1}}{r}+C_{2}, \quad \varphi_{4}^{e}=-\frac{C_{1} C_{2}}{r}+C_{3}
$$

Using Equations (74) and (75), we can present Equations (36) for the external space as

$$
g_{11}^{e}=1+\varepsilon_{e} \frac{C_{1}}{r}+\varepsilon_{e}^{2}\left(\frac{C_{1}}{r}\right)^{2}, \quad g_{22}^{e}=r^{2}, g_{44}^{e}=1+\varepsilon_{e}\left(-\frac{C_{1}}{r}+C_{2}\right)+\varepsilon_{e}^{2}\left(-\frac{C_{1} C_{2}}{r}+C_{3}\right)
$$

Comparing these expressions with Equations (25), we can conclude that $C_{1}=1, C_{2}=C_{3}=0$ and $\varepsilon_{e}=r_{g}$. Thus, the components of the metric tensor for the external space become

$$
g_{11}^{e}=1+\frac{r_{g}}{r}+\left(\frac{r_{g}}{r}\right)^{2}, \quad g_{22}^{e}=r^{2}, g_{44}^{e}=1-\frac{r_{g}}{r}
$$

This result demonstrates a specific feature of the Schwarzchild solution for the external space-it does not include the integration constants which allow us to satisfy the boundary conditions on the sphere surface. This result follows from the form of the coordinate condition used to obtain the solution. Indeed, the Einstein equation, Equation (28), includes $g_{22}^{\prime \prime}$ which is zero if $g_{22}=r^{2}$. So, Equation (28), being initially of the second order, reduces to the equation of the first order, and its solution contains only one integration constant which is found from the asymptotic condition. The second constant that could be used to satisfy the boundary condition for $g_{11}$ is missing and this condition is not sa- 
tisfied in the Schwarzchild solution [8].

Consider the internal space for which, as earlier, assume that $\varepsilon_{i}=\chi \mu c^{2}$. Then, Equations (71) and (73) allow us to find $f_{1}^{i}$ and $\varphi_{1}^{i}$. The regular solutions of these equations are

$$
f_{1}^{i}=\frac{r^{2}}{3}, \quad \varphi_{1}^{i}=\frac{r^{4}}{9}
$$

Thus, the radial component of the metric tensor for the internal space becomes

$$
g_{11}^{i}=1+\frac{\chi \mu c^{2}}{3} r^{2}+\left(\frac{\chi \mu c^{2}}{3}\right)^{2} r^{4}
$$

Matching this expression with the first equation in Equations (76), we can conclude that the boundary condition on the sphere surface $g_{11}^{e}(r=R)=g_{11}^{i}(r=R)$ can be satisfied if $\chi \mu c^{2} R^{3}=3 r_{g}$. Formally, Equation (77) can be used to find $\chi$. Indeed, using Equation (20) for $r_{g}$, we arrive at the following expression for the gravitational constant:

$$
\chi=\frac{6 m G}{R^{3} \mu c^{4}}
$$

If $m$ is specified by Equation (42), then $\chi=\chi_{0}$, where $\chi_{0}$ is specified by Equation (6). However, this not the case for the Schwarzchild solution for which

$$
\begin{aligned}
m & =4 \pi \mu \int_{0}^{R} \sqrt{g_{11}} g_{22} \mathrm{~d} r \\
& =4 \pi \mu \int_{0}^{R} \sqrt{\left[1+\frac{\chi \mu c^{2}}{3} r^{2}+\left(\frac{\chi \mu c^{2}}{3}\right)^{2} r^{4}\right]} r^{2} \mathrm{~d} r \\
& \approx 4 \pi \mu \int_{0}^{R}\left(1+\frac{\chi \mu c^{2}}{6} r^{2}+\frac{\chi^{2} \mu^{2} c^{4}}{18} r^{4}\right) r^{2} \mathrm{~d} r \\
& =\frac{4}{3} \pi \mu R^{3}\left(1+\frac{\chi \mu c^{2} R^{2}}{10}+\frac{\chi^{2} \mu^{2} c^{4} R^{4}}{42}\right)
\end{aligned}
$$

Substituting this result in Equation (78), we arrive at

$$
\chi=\chi_{0}\left(1+\frac{\chi \mu}{10} c^{2} R^{2}+\frac{\chi^{2} \mu^{2}}{42} c^{4} R^{4}\right)
$$

As can be seen, $\chi \neq \chi_{0}$. Moreover, the obtained equation cannot be used to find $\chi$ because $\chi$ is the gravitational constant and cannot depend on $R$ and $\mu$. Thus, the asymptotic analysis of the Schwarzchild solution does not allow us to derive the proper expression for the gravitational constant.

\section{Light Ray Deviation in the Vicinity of Sun}

Having proposed the new metrics in Equations (69), we need to check whether it allows us to predict the experimentally found shift angle which specifies the light ray deviation from the straight trajectory in the vicinity of Sun. For the gravitat- 
ing sphere with radius $R$, this angle can be determined from the following equation [4]:

$$
\alpha=2 I-\pi
$$

in which [9]

$$
I=\int_{R}^{\infty} \sqrt{\frac{g_{11}}{g_{22}\left(\frac{g_{22} g_{44}^{R}}{g_{22}^{R} g_{44}}-1\right)}} d r
$$

and $g_{i i}^{R}=g_{i i}(r=R)$.

Consider the linearized solutions for which

$$
g_{11}=1+f_{1}, \quad g_{22}=r^{2}\left(1+f_{2}\right), \quad g_{44}=1+f_{4}
$$

For the Schwarzchild solution in Equations (76), we have

$$
f_{1}=\frac{r_{g}}{r}, \quad f_{2}=0, f_{4}=-\frac{r_{g}}{r}
$$

and Equation (81) becomes

$$
\begin{aligned}
I & =R \int_{R}^{\infty} \frac{\mathrm{d} r}{r \sqrt{r^{2}-R^{2}}}\left[1-\frac{r^{2} f_{4}^{R}-R^{2} f_{4}}{2\left(r^{2}-R^{2}\right)}\right] \\
& =R \int_{R}^{\infty} \frac{\mathrm{d} r}{r \sqrt{r^{2}-R^{2}}}\left[1+\frac{r_{g}}{2 r}+\frac{r_{g} r}{2 R(r+R)}\right] \\
& =\frac{\pi}{2}+\frac{2 r_{g}}{R}
\end{aligned}
$$

Substitution in Equation (79) yields

$$
\alpha=2 \bar{r}_{g}
$$

in which, as earlier, $\bar{r}_{g}=r_{g} / R$. Calculation for Sun [4] gives the traditional result $\alpha=1.75^{\prime \prime}$ which is in good agreement with the existing experimental data [10].

For the obtained linearized solution in Equations (69),

$$
f_{1}=r_{g}\left(\frac{1}{r}-\frac{3 r^{2}}{5 R^{3}}\right), \quad f_{2}=r_{g}\left(-\frac{1}{2 r}+\frac{3 R^{2}}{10 R^{3}}\right), \quad f_{4}=-\frac{r_{g}}{r}
$$

Substitution in Equation (80) yields

$$
I=R \int_{R}^{\infty} \frac{\mathrm{d} r}{r \sqrt{r^{2}-R^{2}}}\left[1-\frac{r^{2} f_{4}^{R}-R^{2} f_{4}}{2\left(r^{2}-R^{2}\right)}+\frac{1}{2}\left(f_{1}-f_{2}+f_{2}^{R}+f_{4}\right)-\frac{r^{2} f_{2}-R^{2} f_{2}^{R}}{2\left(r^{2}-R^{2}\right)}\right]
$$

The first two terms in this equation are the same that in Equation (83), because function $f_{4}$ is the same in Equations (82) and (85). As can be directly checked, the result of integration of the two last terms in Equation (96) is zero. Thus, the result of integration in Equation (86) is the same that in Equation (83), and the shift angle is specified by Equation (84).

Now, calculate the integral in Equation (80) using the second-order approxi- 
mation of the metric tensor in Equations (69). Omitting rather cumbersome transformations, present the final result which is

$$
\alpha=2 \bar{r}_{g}+\frac{139 \pi-128}{320} \bar{r}_{g}^{2}=2 \bar{r}_{g}+0.955 \bar{r}_{g}^{2}
$$

Taking into account that for Sun $\bar{r}_{g}=4.25 \times 10^{-6}$, we can conclude that the second term is negligible in comparison with the first one, and that the shift angle for the obtained metric coefficients in Equations (69) is specified by the traditional Equation (84) which is in good agreement with experiment.

\section{Conclusion}

As follows from the foregoing analysis, the general relativity theory reduces for low intensity gravitation to the Newton theory only for the empty space. For solids, the linearized equations of the general relativity do not describe gravitation and the second-order asymptotic equations should be applied. For this approximation, the traditional expression for the gravitational constant is found for spherically symmetric static problem under special coordinate condition. The linearized Schwarzchild solution is not reduced to the Newton solution. The obtained solution for the metric tensor of the spherically symmetric empty space yields the traditional result for the angle of light ray deviation in the vicinity of Sun.

\section{Conflicts of Interest}

The authors declare no conflicts of interest regarding the publication of this paper.

\section{References}

[1] Vasiliev, V.V. (1997) Mechanics of Solids, 3, 150-155.

[2] Landau, L.D. and Lifshits, E.M. (1988) Field Theory. Nauka, Moscow. (In Russian)

[3] Singe, J.L. (1960) Relativity: The General Theory. North Holland, Amsterdam.

[4] Weinberg, S. (1972) Gravitation and Cosmology. John Wiley and Sons, Inc., New York.

[5] Vasiliev, V.V. and Fedorov, L.V. (2019) Journal of Modern Physics, 10, 1401-1415. https://doi.org/10.4236/jmp.2019.1012093

[6] Vasiliev, V.V. (2017) Journal of Modern Physics, 8, 1087-1100. https://doi.org/10.4236/jmp.2017.87070

[7] Arroyo-Torres, D., Wittkovski, M., Marcaide, J.M. and Hauschildt, P.H. (2013) Astronomy and Astrophysics, 554, A76. https://doi.org/10.1051/0004-6361/201220920

[8] Vasiliev, V.V. and Fedorov, L.V. (2018) Journal of Modern Physics, 9, 2482-2494. https://doi.org/10.4236/jmp.2018.914160

[9] Logunov, A.A. (2006) Relativistic Theory of Gravitation. Nauka, Moscow. (In Russian)

[10] Froeschle, M., Mignard, F. and Arenou, F. (1997) Determination of the PPN Parameter $\gamma$ with the Hipparcos Data. Proceedings of the ESA Symposium 'Hipparcos Venice 97’ Italy, 13-16 May 1997, ESA SP-402, 49-52. 